Please send trade news information and illustrations to Arveen Bajaj at the $B D J$, Nature Publishing Group, The Macmillan Building, 4-6 Crinan Street, London N1 9XW.

Trade news is provided as a service to readers using text and images from the manufacturer, supplier or distributor and does not imply endorsement by the BDJ. Normal and prudent research should be exercised before purchase or use of any product mentioned.

\section{Scalers from BDSI}

Taking scaling control to another level are these newly designed models equipped

with an LCD digital screen that display the mode and power setting from 1 to 50 and "AGC" Auto Gain Control enabling the user to choose between Scaling, Perio and Endo modes with one touch of a button. The units respond automatically delivering more power to the tip when tackling difficult areas. The Piezo model comes with a newly designed autoclavable titanium piezo hand piece with a 2 year warranty and 4 tips. The tips are compatible with other brands. The Magnetostrictive model is available in 25 and $30 \mathrm{~K}$ and comes with 2 inserts. Both models have a 3-year PC board warranty.

Reader response number 52

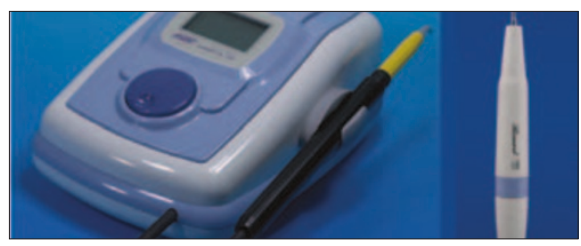

Interactive Patient

\section{Education}

MedVisor dental is the dynamic communication tool that ushers in a new era in patient education. Because it's interactive you can personalise your explanation of diagnoses, treatment options and procedures. Medvisor's custom import/export features enable you to incorporate information unique to each patient. Highly detailed three dimensional animations effectively illustrate complex information. Using pen enabled computers or a graphics tablet on your existing PC you can further reinforce your presentation by drawing upon the animation. All major dental topics are covered and MedVisor is so easy any team member can use it. MedVisor dental the next generation in patient education and treatment planning. Reader response number 53

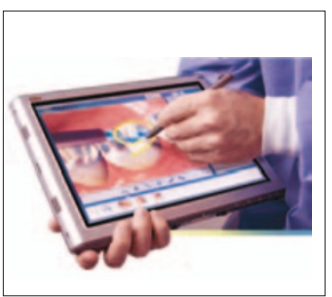

\section{Einstein Intraoral Camera}

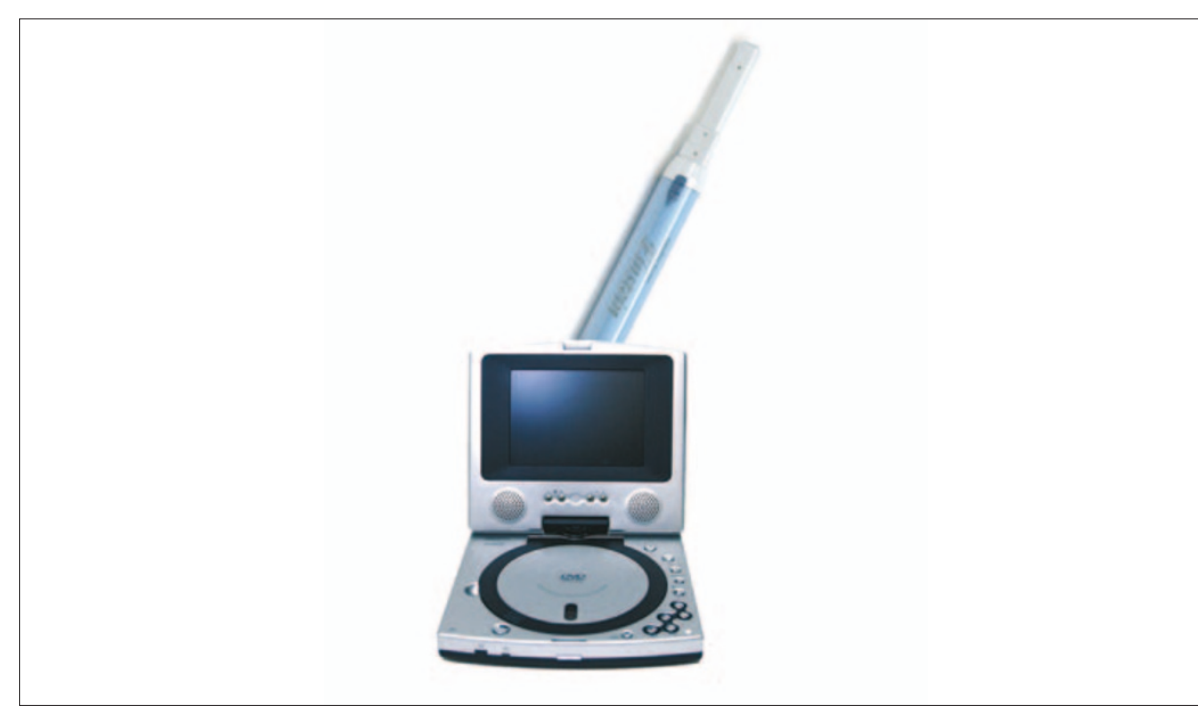

The new high-tech wireless intra-oral camera, Einstein version 2, produces super high quality images with 1.36 mega-pixels CCD and auto focus function. The Einstein 2 images and stores 16 still-images in onboard memory. Equipped with simple ergonomic buttons, operation has never been this easy. It takes both intra-oral and extra-oral images with 8 still-image memory function. You can instantly capture and show the stored images to your patient. captures both intra oral and extra oral
The wireless capability enables you to take close-up shots from various angles as well as positioning the camera to take each tooth in a row. The LED lamps, surrounding the pin lens, maintain original realistic colours. The Einstein camera comes complete with: Copernicus 10inch screen with built in x-ray viewer and camera, Hertz wireless DVD player, bracket for mounting screen on table and as an optional extra a bracket for mounting to a post.

Reader response number 50

\section{Saliva Testing Protecting Your Patients}

GC strongly believes that dentists should use saliva routinely as a diagnostic tool.

It is well known that saliva protects the teeth from demineralisation. When a patient presents with sensitivity, caries or eroded lesions a solution can be found to correct or control these problems when it has been determined why the saliva is not adequately protecting the teeth. Once the problem has been recognised you are able to implement an appropriate prevention and remineralisation programme to rebalance the oral environment thus helping to prevent further effects from demineralisation of the tooth structure. GC's Saliva Testing kit is designed to test the saliva at both resting and stimulated states, as the characteristics of these forms of saliva are different. The GC Saliva Testing Kit has proved to be an extremely and effective powerful tool in assisting with early detection of the condition of the oral environment.

Reader response number 51

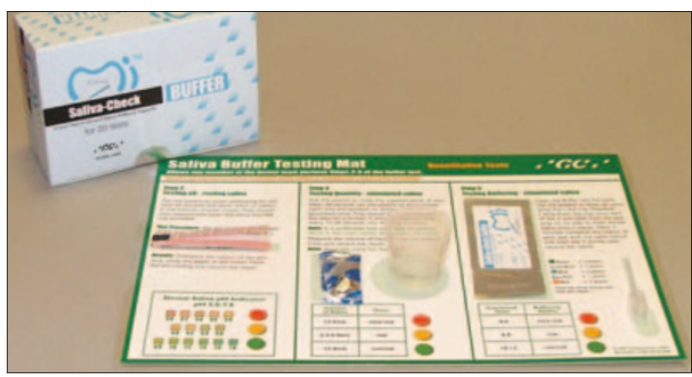



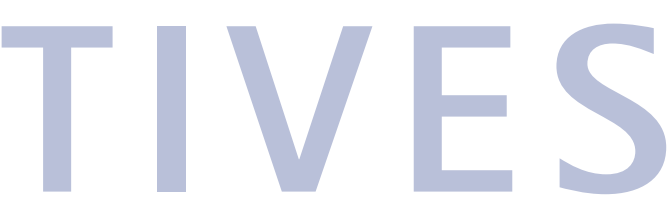

\section{Z100MP lasts 50\% longer}

An innovative change of packaging for Z100MP universal restorative from 3M ESPE has added fifty per cent to its shelf life, extending it to three years. The transition from foil pouches to specially designed capsule bottles protects the material to maintain its superior handling, reduces waste and, because of the smaller size, is easier to store. These package improvements enhance the presentation of this classic composite.

Reader response number 55

\section{M ESPE}

\section{Enhance your}

\section{restorative procedures with Invisalign}

Invisalign is a powerful tool for improving treatment outcomes in a variety of restorative cases. This technology is being used for orthodontic alignment prior to restorative treatment. Invisalign enables practitioners to close spaces and better align teeth prior to finally restoring with veneers, crowns, bridges or implants. The use of Invisalign in these situations means that patients benefit from aesthetic treatment from the outset, without the oral hygiene issues commonly associated with fixed appliances. Invisalign runs one-day course teaching the basics of getting started with Invisalign and includes Invisalign case diagnosis and treatment planning, interactive computer hands-on and practice management and marketing sessions.

Reader response number 56

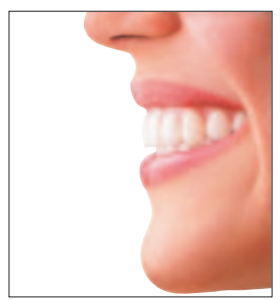

\section{Cyber - Restorative Products for the Future}

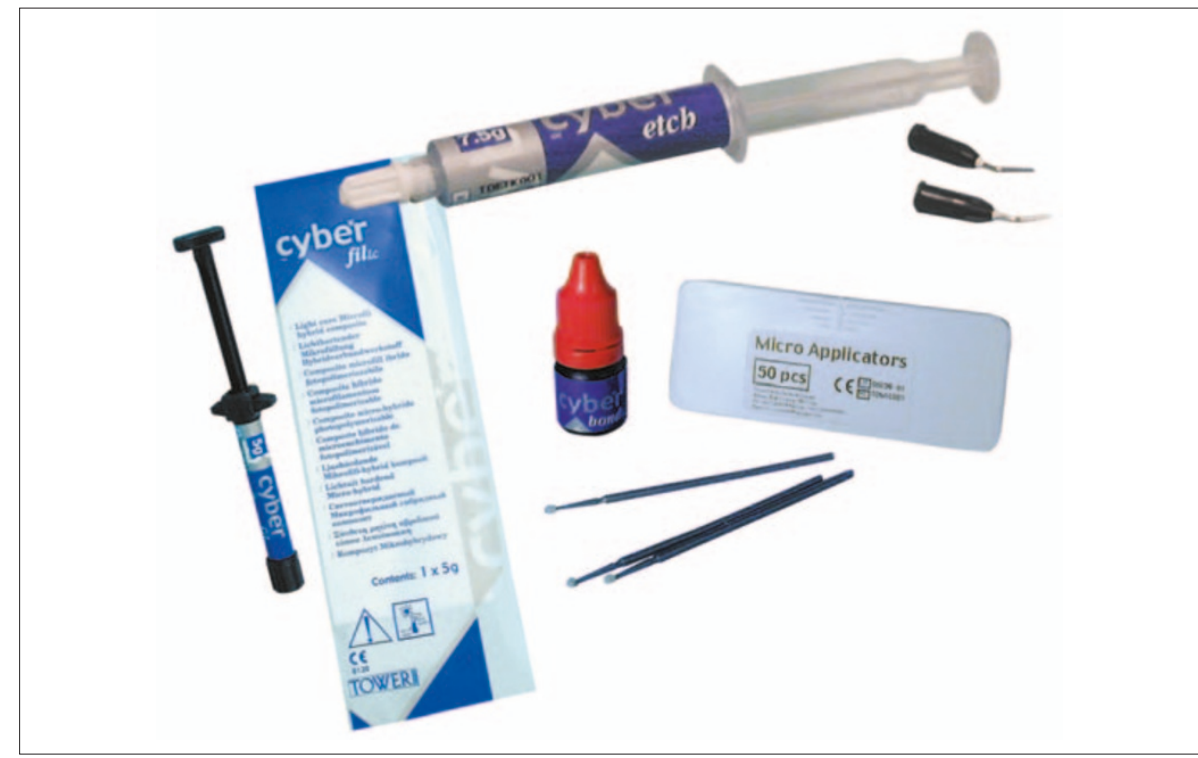

Tower Dental has four new restorative products in their Cyber range. CyberFil is a microfil hybrid composite for anterior and posterior restorations. Strongly radiopaque and light curing, it possesses excellent colour stability and polishes to a glossy and durable finish. It comes in thirteen Vita shades. CyberFil is specially designed for use with CyberEtch gel and CyberBond. CyberEtch is a blue etch gel containing 37\% phosphoric acid. Coloured blue for easy identification on application and removal, it is ideal for the etching of tooth enamel and as a dentine conditioner, prior to bonding direct composite restorations. It is indicated for a wide range of appli-

\section{The new Pegasus Amalgam Well is here}

Astek Innovations are pleased to announce their new Pegasus Amalgam Well which compliments their famous amalgam carriers. The new Pegasus Well is machined from a solid block of stainless steel, carefully bottom weighted to prevent spillages and comes with a handy anti-slip o-ring for even more security. Being fully autoclavable means that this handy well will give years of service and priced at just £12.95 represents excellent value for money. With every 4 Pegasus Amalgam Carriers purchased@ @ust £6.50 each we will offer you a free Pegasus Amalgam Well saving £12.95. Pegasus Amalgam Carriers are available in Straight, 45 and 90 degree angle versions. Reader response number 57

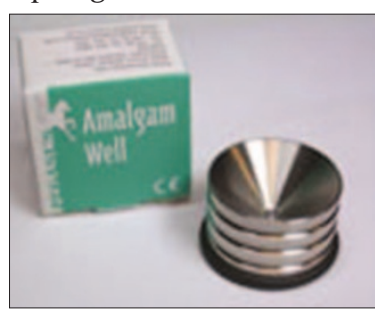




\section{The Direct Approach from GC UK}

You are not unusual if you find that you sometimes compromise your composite aesthetic results by using a single shade of composite for the majority of patients. Help is now at hand with GC Gradia Direct. Due to the shade adaptation of Gradia Direct to the surrounding tooth structure, you will be astounded by the brilliant aesthetics of your restoration, even when you use only one shade. This material will provide you with fantastic aesthetic results due to the unique particle structure and light reflectiveness of Gradia Direct. When dealing with more demanding and complex cases a combination of different shades enables unlimited aesthetic results. GC Gradia Direct offers you the complete assortment from bleach white to dark translucent to outside special shades. Radiopaque GC Gradia Direct Posterior combines aesthetics with excellent mechanical properties by means of high fracture toughness to resist occlusal stress, high wear resistance and low wear on opposing dentition. GC have developed a new shade guide that takes in to account the chroma, hue and value of each composite shade whether you have opted for a single-shade or if you are extensively restoring a tooth and require multiple shades. This shade guide incorporates 2 special shades, which are placed underneath or on top of a standard shade to further enhance your restoration.

Reader response number 58

\section{www.kerrpremise.com}

Premise is the very first highly aesthetic, restorative composite that gives you ultra-low shrinkage (up to $45 \%$ less than other brands currently on the market). Achieving vital, lifelike restorations with low shrinkage has always been a challenge for the dental practitioner, until now. Premise has been introduced by KerrHawe as a complete solution for the aesthetic demands of the more discerning practitioner, who is looking for perfect restorative results every time. Excellent handling in terms of easy sculpability and a non-sticky consistency combined with high aesthetics, superior strength and polishability makes Premise the choice of composite for both anterior and posterior restorations. Using a trimodal approach to nanotechnology, Premise contains three different sized fillers to optimise polish, handling, and strength and to reduce polymerisation shrinkage. Yet there is no complicated shading system to master or special polishing technique to follow. Just choose your shade, place, cure and finish.

Reader response number 59

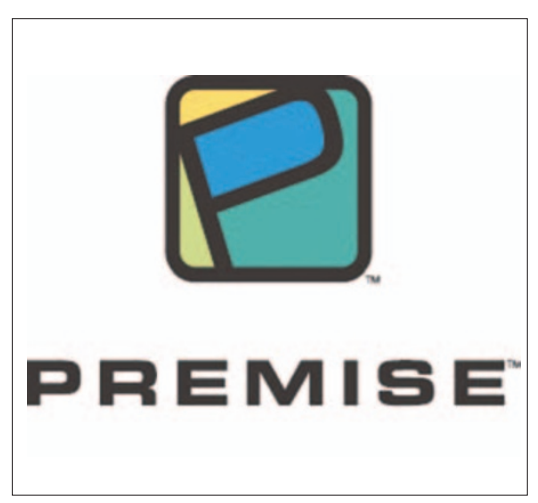

\section{Renamel Microfill}

Renamel Microfill, the only composite ever to receive a five star rating by Reality and its panel of independent evaluators, has now received its 7th consecutive five star award. It has also been the number one rated composite for the past 17 years, and has been awarded the aesthetic Product of the Year for 3 of the past 7 years. Renamel Microfill was the first

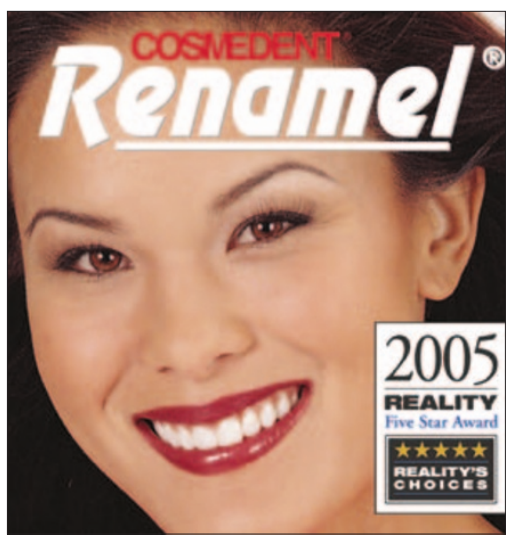
direct resin material designed to mimic natural teeth by simulating enamel. For 20 years, Renamel Microfill has stood above all other composites because it has the best colour range, opacity, easeof-use, handling, remarkably natural appearance and long-term wear-resistance. Additionally, its 25 useful shades (accurately matched to the Vita shade guide) enable dentists to have ultimate control during every restoration. If you want the most lifelike lustre of a natural tooth, the easiest longterm maintenance with the least wear, and the best simulation of natural teeth, then Renamel Microfill is the material for you.

Reader response number 60

\section{Grandio awarded 5}

\section{Stars}

VOCO nano-hybrid composite receives top marks from the Dental Advisor. The committee also granted this product the title 'Editors' Choice'. The clinical evaluation of this product was based on 132 restorations placed in 76 patients. Even one year after the Grandio fillings had been placed in the cavities, they still retained their appearance. After 12 months, the clinical aspects and aesthetics of 94 percent of the fillings remained perfect. Especially the optical properties of Grandio are impressive: the Dental Advisor testers determined that thanks to its translucency, Grandio adapts well to the shade of the adjacent teeth to create optimum aesthetics. No filling was seen to have discoloured. Not only the aesthetics, but also the handling properties were compelling: in spite of its high filler content of $87 \%$ by weight and the resulting physical advantages such as strength, polymerisation shrinkage (1.57\%) and abrasionresistance, nano-composite is very supple, easily contoured and is non-sticky.

Reader response number $\mathbf{6 1}$

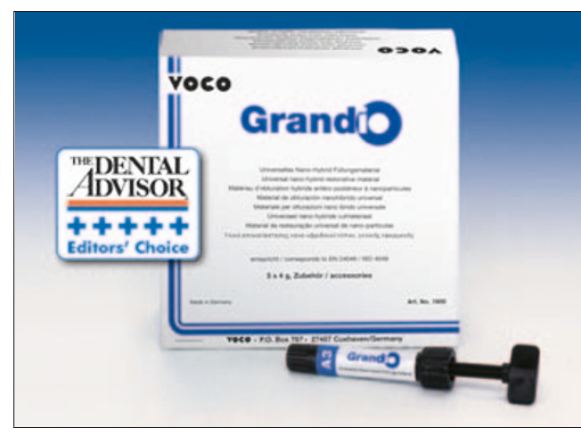

Innovation in Rural England

Many dental team members have been blissfully unaware that there is a hive of industry in rural Kent where some of the world's most highly respected dental products are designed and manufactured. Awareness changed a short while ago though, when Advanced Healthcare Ltd. launched AMALGOMER and AMALGOMER CR, the world's strongest GICs, onto the UK dental market. Prior to launching these branded products AHL were known amongst the dental industry for making high quality materials for other dental companies. AMALGOMER and AMALGOMER CR are a result of British innovation and are based on the reputation of well over 60 million fillings worldwide. AMALGOMER and AMALGOMER CR are the strongest, most durable glass ionomer filling materials available and are the result of many years of GIC development and refinement.

Reader response number 62 